Parapsychology

\title{
MacLab, St Louis, to shut
}

Washington

After six controversial years, the McDonnell Laboratory for Psychical Research at Washington University, St Louis, finally closed at the end of August. The equivocal claims of evidence for extra-sensory perception (ESP) that have emerged from the laboratory have apparently not been sufficient to persuade heirs of the late James McDonnell, a devotee of the paranormal, to continue giving financial support to parapsychology.

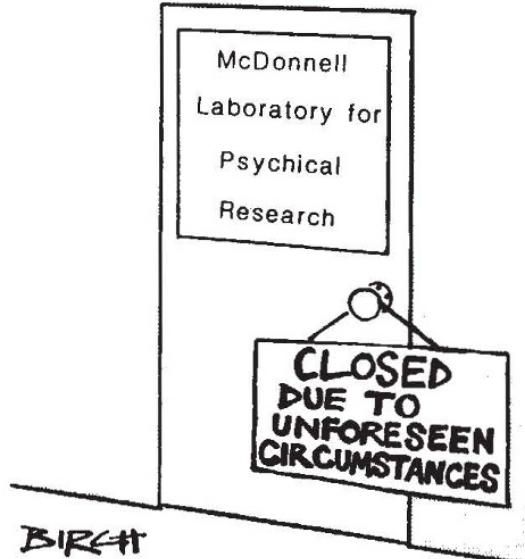

The laboratory, known as MacLab, never fully recovered from the shock of James Randi's Project Alpha in 1983. Randi, a professional magician and foe of sloppy parapsychological research, sent two protégés into MacLab posing as gifted young psychics; the two were investigated at the laboratory for three years before Randi announced the deception at a press conference.

Rancour persists between Randi and the director of MacLab, Peter Phillips. Phillips admits that he was deceived, but says the laboratory never published a claim that it had found psychic abilities, and that he eventually devised experimental protocols that defeated the self-proclaimed psychics. He says Randi used Project Alpha for self-advertisement. Randi, for his part, says Phillips had no idea what was going on until he was persuaded to use protocols supplied by Randi himself; he also says Phillips prepared a printed report claiming psychic effects that was withdrawn once Randi had shown how easy it would be for a magician to cheat.

Since Project Alpha, the research at MacLab has been carried out by a research student Michael Thalbourne. Thalbourne says he has not found any unequivocal, repeatable demonstration of ESP but that evidence for it has surfaced several times in unexpected ways. But according to Randi, Thalbourne's research is still anything but meticulous and Thalbourne has a tendency to re-examine data after the fact for significant effects. $\mathrm{He}$ accuses the experimenters of having a prior conviction that ESP effects are there to be discovered which leads them into error.

The McDonnell Foundation's original bequest (of $\$ 500,000$ ) was intended to run MacLab for only five years, later extended for a further year. Randi says he would

Technostress

\section{Another Japanese discovery}

Tokyo

"TECHNOSTRESS" is a medical condition that will not be found in the textbooks, but which the Japanese are taking seriously indeed they bear responsibility for adding the word to the "English" language. Popular magazines are full of articles on what to do about it and a patent baldness remedy warns that at the very least the "technostress era" will make all your hair fall out. And the Ministry of Health and Welfare has now announced that a full scale study is to be launched to find ways to alleviate it.

Technostress is the stress and concomitant psychosomatic disorder induced by the introduction of high technology. Usually high technology means office automation: it includes the stress produced by working at rhythms dictated by machine, the strain of spending a day looking at a video monitor, and the loss of self-esteem of those who find themselves unable to master new equipment or those who, as one Japanese put it, were "replaced by a thing no bigger than a box of matches".
The study has been launched after several reports of sharp increases in the number of patients who work with or around computers admitted to hospital with psychosomatic disorders. A recent survey carried out at Nihon University also showed that close to half of all visits to general medical practitioners resulted from stress-related disorders.

The first aim of the study will be to try to unravel how stress affects the incidence of disease at different stages of the life cycle. There is already considerable evidence that high blood pressure and ulcers are related to stress but links will also be looked for in disorders such as heart attacks and cancer. And perhaps more important, a ministry official said, is that the study will put an end to thinking of many disorders as being either physical and requiring the treatment by a doctor, or mental and requiring treatment by a psychiatrist. It is hoped that environments that minimize stress can be designed and perhaps counselling services provided that will give advice on how to deal with stressrelated disorders.

Alun Anderson

\section{Indian astronomers to help Soviets}

\section{New Delhi}

INDIA, which has been invited to join the International Halley Watch, has also signed a protocol with the Soviet Union for a joint study of Halley's comet during its appearance in 1985-86. Data from Indian observatories will help Soviet astronomers to make mid-course corrections of the Vega spaceprobes now on their way to meet the comet in mid-March 1986. The protocol also covers collaborative observations from both Indian and Soviet ground stations for polarization measurements, speckle interferometry, and infrared and radio observations at decametre wavelength.

The national programme is being coordinated by the Indian Institute of Astrophysics in Bangalore which is operating the 40 -inch reflector telescope at Kavalur near the east coast in Tamilnadu state. Apart from Kavalur, the programme centres around the Nizamiah Observatory in Hyderabad, which has a 48-inch reflector, and the 40-inch telescope in the solar observatory at Nainital in the western Himalayas. According to Professor K.R. Sivaraman of the Indian Institute of Astrophysics, who is the Indian representative in the International Halley Watch, the unique locations of these observatories was a reason for India being invited to join the international programme. The longitudinal gap between Chinese and Soviet observatories is expected to be bridged by the Indian stations.

According to Professor Sivaraman, the planned observations include astrometry, the comet's spectroscopy and infrared studies. Scintillation studies of radio sources occulted by the comet's tail are planned using the radio telescope at Ooty on the Nilgiri Hills in south India. Another radio telescope at Gouribidanur near Mysore will be used to observe the comet at decametre wavelength. The Tata Institute of Fundamental Research will fly balloons carrying telescopes up to $\mathbf{3 0}$ kilometres height for infrared observations.

K. S. Jayaraman 